\title{
A hanseníase em um distrito administrativo de Belém, estado do Pará, Brasil: relações entre território, socioeconomia e política pública em saúde, 2007-2013
}

\section{Leprosy in an Administrative District of Belém, Pará State, Brazil: relations between territory, socioeconomics, and public health policy, 2007-2013}

\author{
Nelson Veiga Gonçalves ${ }^{1,2}$, Rita Cristina Cotta Alcântara ${ }^{3}$, Alcinês da Silva Sousa Júnior², Alba Lúcia Ribeiro Raithy \\ Pereira $^{4}$, Claudia do Socorro Carvalho Miranda ${ }^{2}$, João Sérgio de Sousa Oliveira ${ }^{2,5}$, Ana Caroline Brasil Viana Melo², \\ Juan Andrade Guedes², Rodrigo Junior Farias da Costa 2 , Simone Beverly Nascimento da Costa ${ }^{6}$, Weber Marcos², \\ Rafaela Pereira Gomes², Rafael Aleixo Coelho de Oliveira², Vera Regina da Cunha Menezes Palácios ${ }^{2}$ \\ ' Universidade Federal Rural da Amazônia, Instituto Ciberespacial, Belém, Pará, Brasil \\ 2 Universidade do Estado do Pará, Centro de Ciências Biológicas e da Saúde, Departamento de Saúde Comunitária, Laboratório de Epidemiologia e \\ Geoprocessamento, Belém, Pará, Brasil \\ ${ }^{3}$ Universidade Federal do Pará, Instituto de Ciências da Saúde, Programa de Pós-Graduação em Saúde, Ambiente e Sociedade na Amazônia, Belém, \\ Pará, Brasil \\ ${ }^{4}$ Universidade do Estado do Pará, Centro de Ciências Biológicas e da Saúde, Departamento de Patologia, Belém, Pará, Brasil \\ 5 Universidade do Estado do Pará, Centro de Ciências Biológicas e da Saúde, Departamento de Ciências do Movimento Humano, Belém, Pará, Brasil \\ - Universidade do Estado do Pará, Centro de Ciências Biológicas e da Saúde, Departamento de Saúde Comunitária, Belém, Pará, Brasil
}

\begin{abstract}
RESUMO
OBJETIVO: Analisar a distribuição espacial da hanseníase em Mosqueiro, o $1^{\circ}$ Distrito Administrativo de Belém, estado do Pará, Brasil. MATERIAIS E MÉTODOS: Estudo transversal e ecológico, utilizando dados do Sistema de Informação de Agravos de Notificação sobre o município de Belém do período de 2007 a 2013, e do Censo 2010 do Instituto Brasileiro de Geografia e Estatística. Foram realizadas análises espaciais das relações entre a taxa de detecção da hanseníase, o índice de condições de vida (ICV) e a cobertura da Estratégia Saúde da Família (ESF). RESULTADOS: A taxa de detecção anual de casos novos apresentou uma tendência decrescente, com um surto em 2009. Contudo, a endemicidade média da doença foi alta. $O$ perfil dos indivíduos mais acometidos foi o gênero masculino (66,67\%), de escolaridade de nível fundamental (66,67\%), da faixa etária de 16 a 59 anos (82,05\%), residente na zona urbana $(74,36 \%)$, com grau zero de incapacidade física $(53,84 \%)$ e classificação operacional multibacilar (76,92\%). A maioria era de casos novos (64,10\%), com entrada por encaminhamento (38,46\%). A distribuição espacial da hanseníase não foi homogênea nos territórios das ESF, apresentando áreas com transmissão ativa da doença, com casos novos multibacilares sem tratamento, padrão muito alto de endemicidade e ICV muito baixo, tais como Maracajá, Carananduba e Baia do Sol. CONCLUSÃO: As análises espaciais, utilizando kernel e álgebras de mapas, foram eficazes para a construção do cenário epidemiológico da hanseníase em Mosqueiro. Ressalta-se a necessidade de expansão da cobertura da ESF, para a melhoria de ações de controle dessa doença produzida sob a lógica perversa das iniquidades sociais.
\end{abstract}

Palavras-chave: Hanseníase; Epidemiologia; Análise Espacial; Estratégia Saúde da Família.

\begin{abstract}
OBJECTIVE: To analyze the spatial distribution of leprosy in Mosqueiro, the $1^{\text {st }}$ Administrative District of Belém, Pará State, Brazil. MATERIALS AND METHODS: Cross-sectional and ecological study, using data from the Information System for Notifiable Diseases (Sinan) of the Municipality of Belém, from 2007 to 2013, and the 2010 Census, from the Brazilian Institute of Geography and Statistics (IBGE). Spatial analysis of relations among the leprosy detection rate, the living conditions index (LCI), and the coverage of Brazil's Family Health Strategy (FHS) were done. RESULTS: The annual incidence rate of new cases showed a decreasing trend, with an outbreak in 2009. However, the average endemicity of the disease was high. The characteristics of the most affected individuals were male (66.67\%), elementary school (66.67\%), 16-59 age group (82.05\%), urban area of residence (74.36\%), zero degree of physical disability (53.84\%), multibacillary form (76.92\%), new cases (64.10\%), and medical referral (38.46\%). Spatial distribution of leprosy was not homogeneous in the FHS territories, presenting areas with active transmission of that disease, with new multibacillary cases without treatment, very high pattern of endemicity and very low $\mathrm{LCl}$, such as Maracajá, Carananduba and Baia do Sol. CONCLUSION: The spatial analysis using kernel and map algebra were effective for constructing the epidemiological scenario of leprosy in Mosqueiro. It is important to emphasize the need of expanding the coverage of FHS in order to improve the control actions of that disease produced under the bad logic of social inequities.
\end{abstract}

Keywords: Leprosy; Epidemiology; Spatial Analysis; Family Health Strategy.

\footnotetext{
Correspondência / Correspondence:

Nelson Veiga Gonçalves

Universidade do Estado do Pará, Centro de Ciências Biológicas e da Saúde, Campus II, Laboratório de Epidemiologia e Geoprocessamento Tv. Perebebuí, 2623. Bairro: Marco - CEP: 66087-670 - Belém, Pará, Brasil - Tel.: +55 (91) 3131-1759. E-mail: nelsoncg2009@gmail.com
} 


\section{INTRODUÇÃO}

A hanseníase ou mal de Hansen (MH) ainda persiste como uma doença infectocontagiosa crônica de evolução lenta, causada pelo Mycobacterium leprae, um bacilo intracelular obrigatório que se aloja na célula de Schwann de nervos periféricos, e que, se não tratada precocemente, leva a sequelas dermatoneurológicas com comprometimentos sensitivos e motores, dentre outros ${ }^{1,2}$.

○ contágio dessa doença ocorre por uma pessoa doente, portadora do bacilo de Hansen na forma multibacilar não tratada, que o elimina para o meio exterior em gotículas orais e nasais, contagiando pessoas suscetíveis ${ }^{3}$. Devido ao padrão de multiplicação do bacilo (12 a 14 dias), a doença progride lentamente. $\bigcirc$ período do aparecimento dos primeiros sinais pode levar, em média, de dois a cinco anos, podendo demorar até $30 \operatorname{anos}^{4,5}$.

A hanseníase tem se mantido como uma doença de grande relevância epidemiológica. De acordo com a Organização Mundial da Saúde, no ano de 2015, foram detectados 210.758 casos novos em todo o mundo, sendo que a Índia e o Brasil apresentaram as maiores notificações, com 127.326 e 26.395 casos, respectivamente ${ }^{6}$.

Nesse cenário epidemiológico, o Brasil apresenta uma distribuição espacial não homogênea da doença, que é influenciada por variáveis socioeconômicas, demográficas e de políticas públicas, nas quais se destacam as condições de vida desfavoráveis das populações humanas e as dificuldades de acesso aos serviços de saúde, fatores esses que estão frequentemente presentes na Região Amazônica, sendo o Pará o quarto Estado brasileiro com maior prevalência da doença ${ }^{7,8,9,10}$.

No município paraense de Belém, como em toda a Região Amazônica, a hanseníase é mais incidente em locais com baixa qualidade de vida. Assim, a doença tem apresentado um padrão de endemicidade alto, evidenciando a necessidade de intensificação de ações de vigilância epidemiológica ${ }^{8,11}$. Nesse contexto, $\circ 1^{\circ}$ Distrito Administrativo - Mosqueiro (DAMOS), que faz parte da área insular do referido Município, tem passado por um processo de ocupação desordenada nas últimas décadas, o qual influenciou na mudança do seu perfil epidemiológico, relacionado a várias doenças infecciosas, como a hanseníase ${ }^{12}$. Contudo, a literatura apresenta poucos estudos sobre a distribuição espacial e temporal dessa doença nesse território.

Considerando essa problemática, a saúde coletiva tem procurado analisar a relação entre variáveis socioeconômicas, políticas públicas e epidemiológicas, por meio do índice de condições de vida (ICV), da cobertura da Estratégia Saúde da Família (ESF) e da taxa de detecção anual de casos novos da hanseníase (TDMH), respectivamente. Esses indicadores são utilizados devido a tais variáveis não ocorrerem de forma homogênea nos territórios brasileiros, expressando assim as suas diferenças e desigualdades.

Nesse contexto, o Ministério da Saúde (MS) tem preconizado ações descentralizadas de prevenção, tratamento e monitoramento para o controle da hanseníase no país, utilizando, para tal, o conceito de território presente nas diretrizes que regem a atuação da $\mathrm{ESF}^{13,14,15}$. A análise espacial de dados epidemiológicos vem sendo utilizada de forma expressiva nas últimas décadas, por possibilitar a caracterização da distribuição geográfica de doenças, seus fatores de risco e suas variáveis condicionantes, que são diferentes nos territórios ${ }^{16}$.

Dessa forma, esta pesquisa analisou a distribuição espacial da hanseníase no DAMOS e sua relação com variáveis socioeconômicas e de políticas públicas em saúde.

\section{MATERIAIS E MÉTODOS}

Este estudo transversal e ecológico foi realizado a partir do levantamento de 39 casos confirmados de hanseníase atendidos pelas ESF, no período de 2007 a 2013, no DAMOS. Foram excluídos os casos notificados fora do referido período e não residentes na área de estudo.

A coleta dos dados epidemiológicos e clínicos (idade, sexo, zona de residência, escolaridade, classificação operacional, avaliação do grau de incapacidade física no momento do diagnóstico, modo de entrada e modo de detecção) foi feita no Sistema de Informação de Agravos de Notificação (Sinan), da Secretaria Municipal de Saúde de Belém (Sesma). Os dados foram georreferenciados em campo, no período de janeiro a outubro de 2016, com coordenadas geográficas obtidas com um dispositivo de captura do Sistema de Posicionamento Global (GPS). Nesse momento, também foi realizado levantamento, de caráter observacional, de dados complementares relacionados a aspectos sociodemográficos.

O indicador da variável socioeconômica foi o ICV, cujos dados dos setores censitários do DAMOS foram obtidos no Censo de 2010, do Instituto Brasileiro de Geografia e Estatística (IBGE). No âmbito deste estudo, a variável FAVELA foi denominada AGLOMERADO.

Para o cálculo do ICV, adaptado dos estudos de Paim et al. ${ }^{17}$ e Queiroga et al. ${ }^{18}$, os indicadores socioeconômicos utilizados foram: RENDA (proporção de chefes de família em domicílios particulares permanentes, com rendimento médio mensal igual ou inferior a dois salários mínimos); EDUCAÇÃO (proporção de pessoas de 10 a 14 anos de idade alfabetizadas); AGLOMERADO (proporção de casas em aglomerado subnormal, em relação ao total de domicílios); RM/Q (razão entre o número médio de moradores por domicílio e o número médio de cômodos servindo de dormitório) e SANEAMENTO (percentagem de domicílios com canalização interna ligada à rede global de abastecimento de água). 
O ICV foi calculado para cada setor censitário do DAMOS, sendo considerados os valores médios de RENDA, AGLOMERADO e RM/Q, que foram dispostos em ordem crescente, e os de EDUCAÇÃO e SANEAMENTO, em ordem decrescente. Em seguida, cada um recebeu uma pontuação, crescente, relativa ao valor de cada indicador. Para a obtenção dos valores de ICV (menor valor de 11 e maior de 72), foi realizada a soma da pontuação dos cinco indicadores de cada setor censitário. Os valores de ICV de cada setor foram agrupados em quartis, para que os mesmos pudessem ser classificados em estratos de condições de vida: alto (de 11 a 25), médio (de 26 a 41), baixo (de 42 a 58) e muito baixo (de 59 a 72). Os menores valores de ICV correspondem a melhores condições de vida.

$\mathrm{O}$ indicador relacionado à variável POLÍTICA PÚBLICA foi a área de cobertura das ESF. Para calcular esse indicador, foi realizado o georreferenciamento em campo, e os limites das áreas de abrangência de cada ESF foram calculados posteriormente pela técnica calculate geometry, na zona do fuso 22S, da projeção Universal Transversa de Mercator (UTM), utilizando-se o software ArcGIS v10.2.

A TDMH, para cada área de cobertura da ESF, foi calculada pela equação: $\mathrm{TDMH}=\left(\mathrm{n}^{\circ}\right.$ de casos novos residentes/população residente) X 100.000 habitantes. $O$ indicador foi classificado de acordo com parâmetros do MS em: hiperendêmico (> 40,0); muito alto $(20,0$ a $39,99)$; alto $(10,00$ a 19,99); médio $(2,00$ a 9,99); e baixo $(<2,00)^{18}$.

A análise da distribuição espacial de densidade dos casos foi realizada com a técnica de interpolação kernel. A relação entre o ICV e a TDMH foi analisada pelas técnicas de álgebra de mapas e unique values, que geraram mapas temáticos e coropléticos, com a utilização do software TerraView v4.0.

perfil epidemiológico foi analisado devido à necessidade de descrever a ocorrência da doença relacionada às características dos indivíduos da área de estudo. A análise foi realizada de forma descritiva e inferencial, utilizando o teste estatístico qui-quadrado de proporção, que possibilitou mensurar as diferenças de proporções entre as diversas variáveis. A interpretação dos testes foi feita de acordo com a convenção científica, com p $<0,05$. Nessas análises, foram utilizados os programas Epi Info $^{\mathrm{TM}}$ v7 e BioEstat v5.0.

No presente estudo, foram adotados os preceitos da Resolução $n^{\circ}$ 466/12 do Conselho Nacional de Saúde, que trata de pesquisa com seres humanos, sendo aprovado em 15 de janeiro de 2016, conforme parecer $n^{\circ}$ 1389215, pelo Comitê de Ética em Pesquisa do Instituto de Ciências da Saúde da Universidade Federal do Pará.

\section{RESULTADOS}

Foram georreferenciados os 39 casos de hanseníase no DAMOS, notificados no Sinan da Sesma no período de 2007 a 2013. Considerando a distribuição temporal da ocorrência dos casos por ESF, foi possível observar que $O$ quadro endêmico da doença manteve-se, de forma geral, estável, sem oscilações expressivas (Tabela 1).

Quanto à TDMH, foi verificada uma tendência decrescente no período do estudo, embora essa não tenha apresentado forte correlação estatística.

Foi observado também um decréscimo da TDMH a partir de 2011 e a ocorrência de um surto da doença em 2009, com um grande aumento do número de casos novos acima da média do período. No DAMOS, de acordo com os parâmetros do $\mathrm{MS}^{19}$, a endemicidade média (1 1,97 casos/100.000 habitantes) foi considerada alta $(10,00$ a 19,99 casos/100.000 habitantes) (Figura 1).

$\mathrm{Na}$ análise da distribuição dos casos, segundo o perfil sociodemográfico, foi possível observar que os maiores percentuais de casos notificados eram de indivíduos do sexo masculino $(66,67 \%)$, de escolaridade de nível fundamental $(66,67 \%)$, da faixa etária de 16 a 59 anos $(82,05 \%)$ e da zona de residência urbana (74,36\%) (Tabela 2).

As variáveis que apresentaram significância estatística $(p<0,05)$ foram sexo masculino ( $p=0,0374)$, escolaridade de ensino fundamental ( $p$ $<0,0001)$, zona de residência urbana ( $p<0,0001)$ e faixa etária de 16 a 59 anos $(p=0,0001)$.

Tabela 1 - Série histórica dos casos de hanseníase notificados nos territórios adscritos pela ESF no DAMOS, estado do Pará, Brasil, entre 2007 e 2013

\begin{tabular}{lccccccccccccccccc}
\hline \multicolumn{1}{c}{ ESF } & \multicolumn{2}{c}{2007} & \multicolumn{2}{c}{2008} & \multicolumn{2}{c}{2009} & \multicolumn{2}{c}{2010} & \multicolumn{2}{c}{2011} & \multicolumn{2}{c}{2012} & \multicolumn{2}{c}{2013} & \multicolumn{2}{c}{ Total } \\
& $N$ & $\%$ & $N$ & $\%$ & $N$ & $\%$ & $N$ & $\%$ & $N$ & $\%$ & $N$ & $\%$ & $N$ & $\%$ & $N$ & $\%$ \\
\hline Aeroporto & 2 & 5,13 & 4 & 10,26 & 1 & 2,56 & 1 & 2,56 & - & - & 2 & 5,13 & 2 & 5,13 & 12 & 30,77 \\
Carananduba & 1 & 2,56 & 1 & 2,56 & 3 & 7,69 & - & - & 4 & 10,26 & 2 & 5,13 & 1 & 2,56 & 12 & 30,77 \\
Maracajá & 1 & 2,56 & - & - & 5 & 12,82 & 2 & 5,13 & - & - & 1 & 2,56 & 1 & 2,56 & 10 & 25,64 \\
Sucurijuquara & 1 & 2,56 & - & - & 1 & 2,56 & - & - & - & - & 1 & 2,56 & - & - & 3 & 7,69 \\
Baia do Sol & - & - & 1 & 2,56 & 1 & 2,56 & - & - & - & - & - & - & - & - & 2 & 5,13 \\
\hline \multicolumn{1}{c}{ Total } & 5 & 12,81 & 6 & 15,38 & 11 & 28,19 & 3 & 7,69 & 4 & 10,26 & 6 & 15,38 & 4 & 10,25 & 39 & 100,00 \\
\hline
\end{tabular}

Teste qui-quadrado para associação $(p=0,2237)$. Sinal convencional utilizado: - Dado numérico igual a zero, não resultante de arredondamento. 


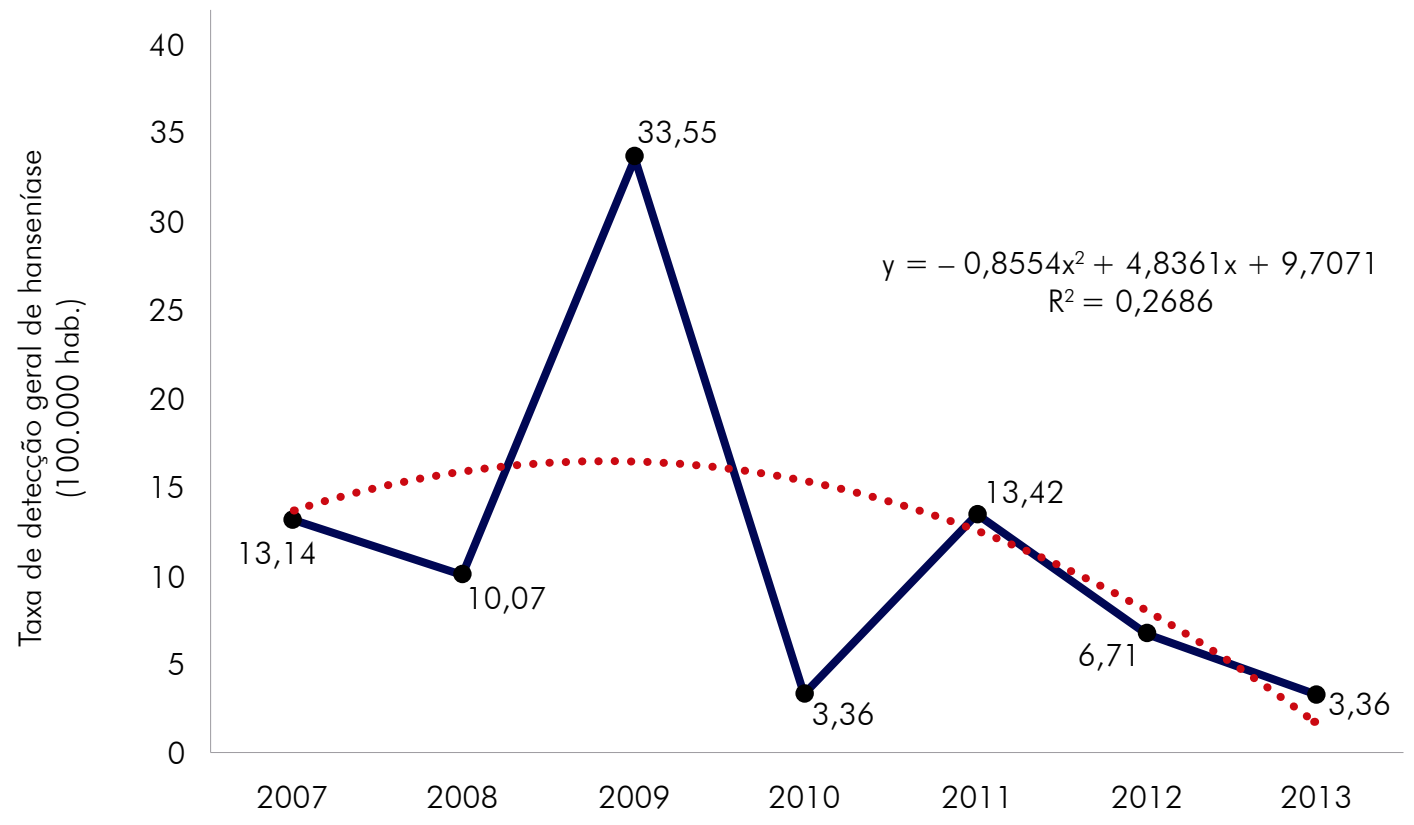

Figura 1 - Taxa de detecção anual de casos novos de hanseníase nos territórios adscritos pela ESF no DAMOS, estado do Pará, Brasil, entre 2007 e 2013

Tabela 2 - Distribuição dos casos notificados, segundo o perfil sociodemográfico, nos territórios adscritos pela ESF no DAMOS, estado do Pará, Brasil, entre 2007 e 2013

\begin{tabular}{|c|c|c|c|}
\hline Variável & Frequência $(N=39)$ & $\%$ & $p^{*}$ \\
\hline \multicolumn{4}{|l|}{ Gênero } \\
\hline Feminino & 13 & 33,33 & \multirow{2}{*}{$0,0374^{\dagger}$} \\
\hline Masculino & 26 & 66,67 & \\
\hline \multicolumn{4}{|l|}{ Escolaridade } \\
\hline Analfabeto & 2 & 5,13 & \multirow{4}{*}{$0,0001^{\dagger}$} \\
\hline Ensino fundamental & 26 & 66,67 & \\
\hline Ensino médio & 5 & 12,82 & \\
\hline Ensino superior & 6 & 15,38 & \\
\hline \multicolumn{4}{|l|}{ Faixa etária (anos) } \\
\hline$\leq 15$ & 1 & 2,56 & \multirow{3}{*}{$0,0001^{\dagger}$} \\
\hline $16-59$ & 32 & 82,05 & \\
\hline$\geq 60$ & 6 & 15,39 & \\
\hline \multicolumn{4}{|l|}{ Zona de residência } \\
\hline Urbana & 29 & 74,36 & \multirow{4}{*}{$<0,0001^{\dagger}$} \\
\hline Periurbana & 8 & 20,52 & \\
\hline Rural & 1 & 2,56 & \\
\hline Sem informação & 1 & 2,56 & \\
\hline
\end{tabular}

*Teste qui-quadrado de Pearson para proporções $(p<0,05) ;{ }^{\dagger}$ Proporções diferem significativamente ao nível de 0,05.

Ao analisar a distribuição das variáveis clínicas dos casos notificados, foi observado que os dados de grau de incapacidade física, classificação operacional, modo de entrada e modo de detecção apresentaram significância estatística $(p<0,05)$, com maior destaque para: detecção de grau zero, com 53,84\%; multibacilar, com 76,92\%; casos novos, com $64,10 \%$; e modo de entrada por encaminhamento, com $38,46 \%$ (Tabela 3).

A expressão visual da ocorrência dos casos notificados de hanseníase, nos territórios estudados, mostrou uma distribuição não homogênea da doença. Em determinadas áreas das ESF Carananduba e Maracajá, foi observada muito alta densidade de casos; nas ESF Aeroporto e Sucurijuquara, alta densidade de casos; e na ESF Baia do Sol, média densidade de casos.

Com relação à taxa de cobertura territorial das ESF no DAMOS, foi observado que aproximadamente 53\% desse Distrito Administrativo não está coberto pela política pública, sendo que, nas referidas áreas, a maior expressividade foi de casos multibacilares (Figura 2).

Na análise dos territórios das ESF, foi observado que todos apresentaram áreas com transmissão ativa de 
hanseníase, relacionadas principalmente pela entrada de casos novos multibacilares sem tratamento e a ocorrência da doença em menor de 15 anos de idade. Os territórios das ESF apresentaram apenas padrões alto e muito alto de endemicidade. Foi observado também que as áreas de ESF com padrão de endemicidade alto estavam relacionadas espacialmente com o ICV muito baixo, como Maracajá, Carananduba e Baia do Sol, sendo que as duas primeiras apresentaram os maiores focos de transmissão ativa e a última, a mais baixa ocorrência da doença (Figura 3).

Tabela 3 - Distribuição dos casos notificados, segundo o perfil clínico, nos territórios adscritos pela ESF no DAMOS, estado do Pará, Brasil, entre 2007 e 2013

\begin{tabular}{|c|c|c|c|}
\hline Variável & Frequência $(N=39)$ & $\%$ & $p^{*}$ \\
\hline \multicolumn{4}{|c|}{ Grau de incapacidade física } \\
\hline Grau zero & 21 & 53,84 & \multirow{4}{*}{$0,0002^{\dagger}$} \\
\hline Grau 1 & 9 & 23,08 & \\
\hline Grau II & 2 & 5,13 & \\
\hline Não avaliado & 7 & 17,95 & \\
\hline \multicolumn{4}{|l|}{ Classificação operacional } \\
\hline Multibacilar & 30 & 76,92 & \multirow{2}{*}{$0,0008^{\dagger}$} \\
\hline Paucibacilar & 9 & 23,08 & \\
\hline \multicolumn{4}{|l|}{ Modo de entrada } \\
\hline Caso novo & 25 & 64,10 & \multirow{3}{*}{$<0,0001^{\dagger}$} \\
\hline Transferência & 12 & 30,77 & \\
\hline Recidiva & 2 & 5,13 & \\
\hline \multicolumn{4}{|l|}{ Modo de detecção } \\
\hline Encaminhamento & 15 & 38,46 & \multirow{4}{*}{$0,0109^{\dagger}$} \\
\hline Demanda espontânea & 8 & 20,51 & \\
\hline Exame de contatos & 2 & 5,13 & \\
\hline Sem informação & 14 & 35,90 & \\
\hline
\end{tabular}

* Teste qui-quadrado de Pearson para proporções $(p<0,05)$; † Proporções diferem significativamente, ao nível de 0,05.
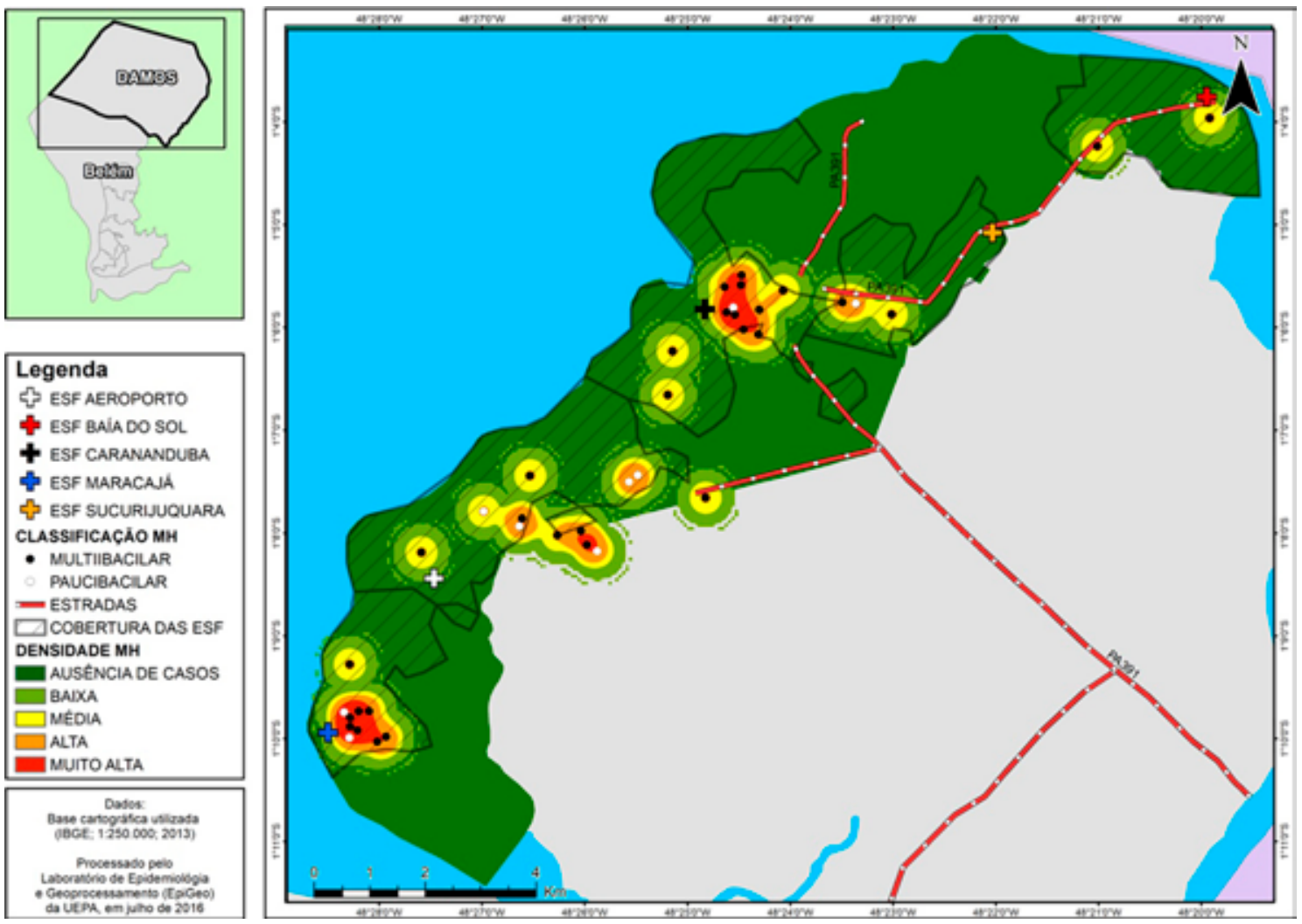

Fonte: EPIGEO/DSCM/CCBS/UEPA, 2016.

Figura 2 - Distribuição espacial da densidade de casos de hanseníase notificados nos territórios adscritos pela ESF no DAMOS, estado do Pará, Brasil, segundo a classificação operacional, entre 2007 e 2013 

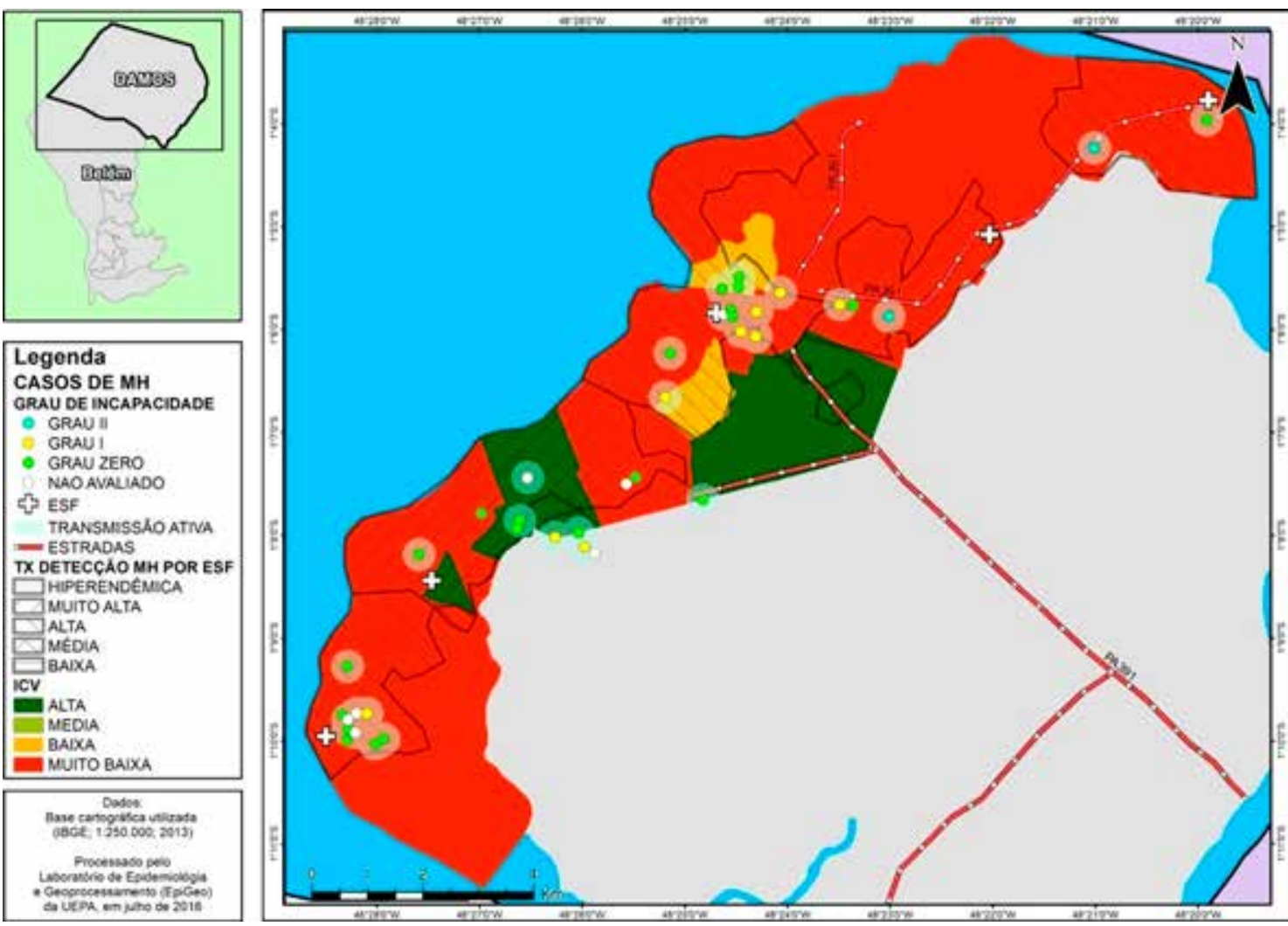

Fonte: EPIGEO/DSCM/CCBS/UEPA, 2016

Figura 3 - Distribuição espacial da relação entre a taxa de detecção da hanseníase e o ICV nos territórios adscritos pela ESF no DAMOS, estado do Pará, Brasil, entre 2007 e 2013

\section{DISCUSSÃO}

A distribuição temporal do número de casos notificados, por ESF, no período de estudo, não apresentou, de forma geral, alterações significativas. Esse fato pode estar relacionado à baixa cobertura dessa política pública no DAMOS. Considerando que a ESF preconiza ações de busca ativa e vigilância de contatos, a ausência desses serviços pode acarretar - estabelecimento de áreas silenciosas. Situações semelhantes foram encontradas por Lana et al. ${ }^{20}$ e Lobato et al. ${ }^{21}$, ao realizarem estudos no Vale do Jequitinhonha, no estado de Minas Gerais, e no município de Igarapé-Açu, no estado do Pará, respectivamente.

A tendência da série temporal mostrou mudanças no padrão de distribuição das taxas de detecção da hanseníase, com decréscimo das mesmas. Embora, essa tendência não tenha apresentado uma forte correlação estatística, sugere a necessidade de maior investigação da ocorrência da doença na área de estudo, pois as mudanças desses padrões podem estar relacionadas a problemas na atenção primária à saúde, resultando na existência de áreas descobertas e consequente subnotificação de $\operatorname{casos}^{22,23,24}$.

No DAMOS, a TDMH apresentou um padrão de alta endemicidade da doença, mostrando, dessa forma, que a hanseníase é um grande problema de saúde pública em Belém, situação essa também observada em vários municípios brasileiros ${ }^{23,25}$. Essa taxa apresentou tendência decrescente no período estudado, fato que pode ser justificado pela intensificação das ações de controle da hanseníase realizadas por órgãos de saúde estadual e municipal, com o apoio do MS, no intuito de eliminar a doença nesse município. As campanhas tiveram grande importância no processo de controle da hanseníase no território brasileiro ${ }^{24}$.

Foi observado, também na série histórica, que ocorreu um surto da doença na área de estudo em 2009. Tal fato pode ser explicado pela realização de campanhas de busca ativa de casos, tratamento da doença e descentralização de ações educativas que ocorreram no Município pelas ESF nesse período. A execução dessas ações, em outros municípios brasileiros, apresentou resultados similares ao observado no DAMOS24,26.

No que diz respeito às variáveis relacionadas ao indivíduo, foi observado que o gênero masculino apresentou o maior percentual de casos $(66,67 \%)$, fato observado também em outras regiões do Brasil e do mundo 27,28,29,30,31. Contudo, a literatura apresenta divergências em relação à prevalência da hanseníase segundo o gênero; em relação ao masculino, alguns autores afirmam que a maior exposição a fatores de risco socioeconômicos, a menor preocupação com a saúde física e a falta de políticas públicas podem estar contribuindo para o diagnóstico tardio, o que poderia justificar o predomínio do gênero feminino em alguns estudos 32,33 . 
Foi observado que o maior percentual de casos ocorreu em pessoas com menor grau de escolaridade, fato que pode estar relacionado à vulnerabilidade socioeconômica em que os casos estão inseridos. Essa relação aponta para a produção socioeconômica da doença que, historicamente, tem sido observada em diversos territórios brasileiros ${ }^{5,28,34,35}$.

Em relação à faixa etária, a doença acometeu indivíduos economicamente ativos, ou seja, de 16 a 59 anos. De maneira geral, o achado corrobora outros estudos, embora considerem diferentes faixas etárias ${ }^{28,30}$. $\bigcirc$ adoecimento dessa parcela da população pode ter impactos na economia do DAMOS, visto que os indivíduos estão sujeitos a desenvolverem incapacidades para a atividade produtiva, gerando um custo social ${ }^{29}$.

Foi verificado também que a hanseníase possui um perfil endêmico urbano, com 74,36\% dos casos notificados no DAMOS, o que pode estar relacionado aos aglomerados urbanos que favorecem a transmissão da doença, acometendo indivíduos em condições sociais menos favorecidas, sem acesso a saneamento básico, com baixa escolaridade, alimentação deficiente e residentes em moradias com condições precárias.

Em relação à classificação operacional da hanseníase, a análise mostrou que os casos multibacilares foram predominantes em áreas cobertas ou não pelas ESF. Esse fato pode estar associado à detecção tardia da doença, que contribui com a manutenção da cadeia de transmissão da mesma, além de casos que poderão desenvolver lesão neural. No que diz respeito aos graus de incapacidade física, foi observado que o maior número de casos ocorreu no grau zero $(53,84 \%)$, seguido por grau | e || $(28,21 \%)$ e não avaliados $(17,95 \%)$, sugerindo problemas operacionais na notificação adequada dos casos, como incompletude e inconsistência de dados $^{35}$.

A análise da correlação entre a alta densidade de casos multibacilares e a distribuição dos graus de incapacidade I e II indicou uma ineficiência da vigilância epidemiológica precoce da hanseníase, que se expressou de forma diferenciada nas áreas de cobertura das ESF no DAMOS, com indicação de diagnóstico tardio nas áreas das ESF Sucurijuquara, Carananduba e Baia do Sol. Observação similar foi realizada por Palácios et al. ${ }^{36,37,38}$, quando analisaram a doença no Pará e mostraram que os municípios estudados refletiam o perfil da classificação operacional do Estado.

Em relação ao modo de entrada, a maioria das notificações apresentou casos novos, seguidos de casos de transferência (doentes que foram transferidos de unidade de saúde) e casos de recidiva, indicando que a transmissão da hanseníase está ativa, ao se observar a prevalência da mesma na área e período de estudo ${ }^{39}$
Considerando que a maior parte do modo de detecção de casos notificados ocorreu por encaminhamento e demanda espontânea (58,97\%) e que a menor foi por exame de contato $(5,13 \%)$, ficou caracterizada a ocorrência de formas passivas de detecção, ou seja, o usuário é quem procurou o serviço de saúde e, a partir disso, obteve o diagnóstico, indicando busca ativa de casos insatisfatória ${ }^{8,13,40,41}$.

Foi observada uma distribuição não homogênea da hanseníase, com aglomeração de casos na área de estudo, o que pode estar correlacionado à ocorrência de maior densidade de casos em áreas com cobertura de ESF, devido às mesmas oferecerem serviços de saúde como diagnóstico e tratamento. No que se refere à observação de uma expressiva área sem cobertura de ESF (53\%), sugere-se a necessidade de expansão dessa política pública, pois há evidências de silêncio epidemiológico da doença na região ${ }^{35,42}$.

A análise espacial da relação entre o ICV e a TDMH mostrou que, nas áreas das ESF de Maracajá e de Carananduba, o ICV foi considerado muito baixo e a taxa de detecção da hanseníase foi alta, inclusive com áreas de transmissão ativa da doença, - que seria justificado pelas condições precárias de vida das populações que moram nessas áreas. Dentre essas condições, a principal foi o adensamento ou aglomerado habitacional, com muitas pessoas coabitando o mesmo domićlio, o que facilita a transmissão da doença. Foi observado também que, nesses territórios, ocorrem grandes percentuais de áreas descobertas pela ESF, o que implica numa possível prevalência oculta da doença e na produção social da mesma $^{35,41,42}$.

\section{CONCLUSÃO}

Foi constatado que a hanseníase no DAMOS ocorreu de forma diferenciada nos territórios adscritos pelas ESF, com decréscimo da doença no período do estudo. Além disso, observou-se uma alta densidade de casos da doença nas áreas com menores ICV e com uma alta taxa de detecção de casos novos, inclusive com classificação operacional multibacilar.

O cenário observado, no presente estudo, pode estar condicionado às questões socioeconômicas, clínicas, epidemiológicas e de políticas públicas, cujas variáveis estão em constante processo de alteração. Desta forma, estudos que consideram a distribuição da doença nos territórios, ao evidenciar as baixas condições de vida das populações, devem ter caráter contínuo e sistemático, pois podem contribuir para a saúde pública.

As análises espaciais dessas variáveis foram eficazes para a construção do cenário epidemiológico da hanseníase na área e período de estudo, as quais poderão servir para processos de tomadas de decisão na vigilância epidemiológica da doença. Dado o exposto, ressalta-se a necessidade de expansão da área de cobertura das ESF no DAMOS, no intuito da 
melhoria de ações de controle e mitigação da doença, que se produz sob a lógica perversa das iniquidades sociais.

\section{APOIO FINANCEIRO}

Agradecemos o apoio logístico e orçamentário do Laboratório de Epidemiologia e Geoprocessamento (EPIGEO) do Departamento de Saúde Comunitária (DSCM) e da direção do Centro de Ciências Biológicas e da Saúde (CCBS) da Universidade do Estado do Pará (UEPA); e o apoio logístico da Sesma.

\section{CONFLITOS DE INTERESSE}

Este trabalho não apresentou conflitos de interesse.

\section{CONTRIBUIÇÃO DOS AUTORES}

Todos os autores contribuíram com a idealização do estudo, a análise e a interpretação dos dados e com a redação do manuscrito, aprovando a versão final publicada. Declaram-se responsáveis pelo conteúdo integral do artigo, garantindo sua precisão e integridade.

\section{REFERÊNCIAS}

1 Britton WJ, Lockwood DNJ. Leprosy. Lancet. 2004 Apr;363(9416):1209-19.

2 Lockwood DN, Saunderson PR. Nerve damage in leprosy: a continuing challenge to scientists, clinicians and service providers. Int Health. 2012 Jun;4(2):77-85.

3 Veronesi R, Focaccia R. Tratado de infectologia. 5. ed. São Paulo: Atheneu; 2015.

4 Ministério da Saúde (BR). Secretaria de Políticas de Saúde. Departamento de Atenção Básica. Guia para o controle da hanseníase. Brasília: Ministério da Saúde; 2002. 89 p. (Série A. Normas e manuais técnicos; n. 111).

5 Barreto JG, Bisanzio D, Guimarães LS, Spencer JS, Vazquez-Prokopec GM, Kitron U, et al. Spatial analysis spotlighting early childhood leprosy transmission in a hyperendemic municipality of the Brazilian Amazon region. PLoS Negl Trop Dis. 2014 Feb;8(2):e2665.

6 World Health Organization. Department of Control of Neglected Tropical Diseases. Global leprosy update, 2015: time for action, accountability and inclusion. Wkly Epidemiol Rec. 2016 Sep;91 (35):405-20.

7 Silva DRX, Ignotti E, Souza-Santos R, Hacon SS. Hanseníase, condições sociais e desmatamento na Amazônia brasileira. Rev Panam Salud Publica [Internet]. 2010 abr [citado 2017 mar 21];27(4):268-75. Disponível em: http://www. scielosp.org/pdf/rpsp/v27n4/a05v27n4.pdf.

8 Ministério da Saúde (BR). Secretaria de Vigilância em Saúde. Sistema nacional de vigilância em saúde: relatório de situação: Pará [Internet]. 5. ed. Brasília: Ministério da Saúde; 2011 [citado 2017 mar 21]. (Série C. Projetos, programas e relatórios). Disponível em: http://bvsms.saude.gov.br/bvs/ publicacoes/sistema_nacional_vigilancia_saude pa_5ed.pdf.

9 Costa LG, Cortela D, Soares RC, Ignotti E. Factors associated with the worsening of the disability grade during leprosy treatment in Brazil. Lepr Rev. 2015 Sep;86(3):265-72.
10 Nobre ML, Dupnik KM, Nobre PJL, Souza MCF, Duppre NC, Sarno EN, et al. Human migration, railways and the geographic distribution of leprosy in Rio Grande do Norte State - Brazil. Lepr Rev. 2015 Dec;86(4):335-44

11 Lanza FM, Lana FCF. Descentralização das ações de controle da hanseníase na microrregião de Almenara, Minas Gerais. Rev Latino-Am Enfermagem. 2011 jan-fev; 19(1):187-94.

12 Prefeitura Municipal de Belém (Pará). $\bigcirc$ processo de revisão do plano diretor do município de Belém. [Internet]. Belém: PMB; 2009 [citado 2017 mar 21]. Disponível em: http://www.belem.pa.gov.br/ planodiretor/.

13 Ministério da Saúde (BR). Secretaria de Políticas de Saúde. Departamento de Atenção Básica. Controle da hanseníase na atenção básica: guia prático para profissionais da equipe de saúde da família. Brasília: Ministério da Saúde; 2001. 84 p. (Série A. Normas e manuais técnicos, n. 1111$)$

14 Ministério da Saúde (BR). Secretaria de Atenção à Saúde. Departamento de Atenção Básica. Política nacional de atenção básica. Brasília: Ministério da Saúde; 2012. 108 p. (Série E. Legislação em saúde).

15 Chaptini C, Marshman G. Leprosy: a review on elimination, reducing the disease burden, and future research. Lepr Rev. 2015 Dec;86(4):307-15.

16 Bakker MI, Scheelbeek PFD, Van Beers SM. The use of GIS in leprosy control. Lepr Rev. 2009 Sep;80(3):327-31.

17 Paim JS, Silva LMV, Costa MCN, Prata PR, Lessa I. Desigualdades na situação de saúde do município de Salvador e relações com as condições de vida. R Ci Med Biol. 2003 jan-jun;2(1):30-9.

18 Queiroga RPF, Sá LD, Nogueira JA, Lima ERV, Silva ACO, Pinheiro PGOD, et al. Distribuição espacial da tuberculose e a relação com condições de vida na área urbana do município de Campina Grande - 2004 a 2007. Rev Bras Epidemiol. 2012 mar; 15(1):222-32. 
19 Ministério da Saúde (BR). Secretaria de Vigilância em Saúde. Departamento de Vigilância das Doenças Transmissíveis. Diretrizes para vigilância, atenção e eliminação da hanseníase como problema de saúde pública: manual técnico-operacional. Brasília: Ministério da Saúde; 2016. $58 \mathrm{p}$.

20 Lana FCF, Amaral EP, Franco MS, Lanza FM. Detecção da hanseníase no Vale do Jequitinhonha - Minas Gerais: redução da tendência epidemiológica ou problemas operacionais para o diagnóstico? Hansen Int [Internet]. 2004 dez [citado 2017 jul 10];29(2):118-23. Disponível em: http://periodicos.ses.sp.bvs.br/pdf/hi/v29n2/ v29n2a05.pdf.

21 Lobato DC, Neves DCO, Xavier MB. Avaliação das ações da vigilância de contatos domiciliares de pacientes com hanseníase no Município de Igarapé-Açu, estado do Pará, Brasil. Rev Pan-Amaz Saude [Internet]. 2016 mar [citado 2017 set 10];7(1):45-53. Disponível em: http://scielo.iec. pa.gov.br/pdf/rpas/v7n1/v7nla06.pdf.

22 Barreto JG, Guimarães LS, Frade MAC, Rosa PS, Salgado CG. High rates of undiagnosed leprosy and subclinical infection amongst school children in the Amazon Region. Mem Inst Oswaldo Cruz. 2012 Dec;107 Suppl 1:60-7.

23 Brito KKG, Andrade SSC, Santana EMF, Peixoto VB, Nogueira JA, Soares MJGO. Análise epidemiológica da hanseníase em um estado endêmico do nordeste brasileiro. Rev Gaucha Enferm [Internet]. 2015 [citado 2017 mar 22];36(no. Esp.):24-30. Disponível em: http:// www.scielo.br/pdf/rgenf/v36nspe/0102-6933-rgenf36-spe-0024.pdf.

24 Monteiro LD, Martins-Melo FR, Brito AL, Alencar $\mathrm{CH}$, Heukelbach J. Padrões espaciais da hanseníase em um estado hiperendêmico no Norte do Brasil, 2001-2012. Rev Saude Publica [Internet]. 2015 dez [citado 2017 mar 21];49:84. Disponível em: http:// www.scielosp.org/pdf/rsp/v49/pt 0034-8910rsp-S0034-89102015049005866.pdf.

25 Ministério da Saúde (BR). Secretaria de Vigilância em Saúde. Situação epidemiológica da hanseníase no Brasil - análise de indicadores selecionados na última década e desafios para eliminação. Bol Epidemiol [Internet]. 2013 [citado 2017 mar 21];44(11):1-12. Disponível em: http:// portalarquivos.saude.gov.br/images/pdf/2014/ junho/11/BE-2013-44--11----Hanseniase.pdf.

26 Passos CEC, Silva AR, Gonçalves EGR, Neiva FGC, Monteiro SG. Hanseníase no Estado do Maranhão: análise das estratégias de controle e os impactos nos indicadores epidemiológicos. Hygeia [Internet]. 2016 jun [citado 2017 mar 21];12(22):88-100. Disponível em: http://www.seer.ufu.br/index.php/ hygeia/article/view/30888.
27 Ramos JM, Martínez-Martín M, Reyes F, Lemma D, Belinchón I, Gutiérrez F. Gender differential on characteristics and outcome of leprosy patients admitted to a long-term care rural hospital in South-Eastern Ethiopia. Int J Equity Health. 2012 Oct; 1 1:56.

28 Barbosa DRM, Almeida MG, Santos AG. Características epidemiológicas e espaciais da hanseníase no Estado do Maranhão, Brasil, 2001 2012. Medicina (Ribeirão Preto) [Internet]. 2014 mar [citado 2017 mar 22];47(4):347-56. Disponível em: http://revista.fmrp.usp.br/2014/vol47n4/REV Caracteristicas-epidemiologicas-e-espaciais-dahanseniase-no-Estado-do-Maranhao.pdf.

29 Lopes VAS, Rangel EM. Hanseníase e vulnerabilidade social: uma análise do perfil socioeconômico de usuários em tratamento irregular. Saude Debate [Internet]. 2014 out-dez [citado 2017 mar 22];38(103):817-29. Disponível em: http://www.scielo.br/pdf/sdeb/v38n103/01031104 -sdeb-38-103-0817.pdf.

30 Negrão GN, Vieira IR, Katayama EMY, Borecki MT. Variáveis epidemiológicas intervenientes na ocorrência da hanseníase no município de Guarapuava, PR. Geografia (Londrina) [Internet]. 2016 jul-dez [citado 2017 mar 22];25(2): 110-29. Disponível em: http://www.uel.br/revistas/uel/index. php/geografia/article/viewFile/24422/20545.

31 Sarmento APA, Pereirao AM, Ribeiro F, Castro JL, Almeida MB, Ramos NM. Perfil epidemiológico da hanseníase no período de 2009 a 2013 no município de Montes Claros (MG). Rev Soc Bras Clin Med [Internet]. 2015 jul-set [citado 2017 mar 22]; 13(3):180-4. Disponível em: http://files.bvs.br/ upload/S/1679-1010/2015/v13n3/a5389.pdf.

32 Varkevisser CM, Lever P, Alubo O, Burathoki K, Idawani C, Moreira TMA, et al. Gender and leprosy: case studies in Indonesia, Nigeria, Nepal and Brazil. Lepr Rev. 2009 Mar;80(1):65-76.

33 Brito $\mathrm{AL}$, Monteiro LD, Ramos Junior AN, Heukelbach J, Alencar CH. Tendência temporal da hanseníase em uma capital do Nordeste do Brasil: epidemiologia e análise por pontos de inflexão, 2001 a 2012. Rev Bras Epidemiol. 2016 jan-mar; 19(1): 194-204.

34 Murto C, Chammartin F, Schwarz K, Costa LMM, Kaplan C, Heukelbach J. Patterns of migration and risks associated with leprosy among migrants in Maranhão, Brazil. PLoS Negl Trop Dis. 2013 Sep;7(9):e2422.

35 Vieira GD, Aragoso I, Carvalho RMB, Sousa CM. Hanseníase em Rondônia: incidência e características dos casos notificados, 2001 a 2012. Epidemiol Serv Saude [Internet]. 2014 jun [citado 2017 mar 22];23(2):269-75. Disponível em: http://www.scielo.br/pdf/ress/v23n2/1679-4974ress-23-02-00269.pdf. 
36 Palácios VRCM, Dias RS, Neves DCO. Estudo da situação da hanseníase no estado do Pará. Rev Para Med [Internet]. 2010 abr-jun [citado 2017 mar 22];24(2):49-56. Disponível em: http://files. bvs.br/upload/S/0101-5907/2010/v24n2/a2122. pdf.

37 Palácios VRCM, Bichara CNC, Silva Junior JB, Dias RS, Gonçalves NV. Leprosy and pregnancy in the state of Pará: an epidemiological perspective. Rev Soc Bras Med Trop [Internet]. 2013 Jul-Aug [cited 2017 Mar 23];46(4):453-60. Available from: http://www.scielo.br/scielo.php? script=sci_arttext\&pid $=$ S0037-86822013000400 453\&lng $=$ en.

38 Palácios VRCM, Bichara CNC, Andriolo RB, Fonteles MJP, Andriolo BNG, Gonçalves NV, et al. Leprosy and pregnancy: a epidemiological and social study in the Pará State, north of Brazil. In: Kopp RS, editor. Leprosy: epidemiology, treatment strategies and current challenges in research. New York: Nova Science Publishers; 2014. Chapter 3; p. $51-70$.

39 Melão S, Blanco LFO, Mounzer N, Veronezi CCD, Simões PWTA. Perfil epidemiológico dos pacientes com hanseníase no extremo sul de Santa Catarina, no período de 2001 a 2007. Rev Soc Bras Med Trop [Internet]. 2011 jan-fev [citado 2017 mar 23];44(1):79-84. Disponível em: http:// www.scielo.br/scielo.php?script=sci_arttext\&pid.
40 Ministério da Saúde (BR). Secretaria de Vigilância em Saúde. Departamento de Vigilância Epidemiológica. Manual de prevenção de incapacidades. 3. ed. Brasília: Ministério da Saúde; 2008. 135 p. (Série A. Normas e manuais técnicos. Cadernos de prevenção e reabilitação em hanseníase; n. 1).

41 Pieri FM, Ramos ACV, Crispim JA, Pitiá ACA, Rodrigues LBB, Silveira TRS, et al. Fatores associados às incapacidades em pacientes diagnosticados de hanseníase: um estudo transversal. Hansen Int [Internet]. 2012 [citado 2017 mar 22];37(2):22-30. Disponível em: http:// www.ilsl.br/revista/detalhe_artigo.php?id=12010\#.

42 Silva MS, Silva EP, Monteiro FF, Teles SF. Perfil clínico-epidemiológico da hanseníase no estado do Acre: estudo retrospectivo. Hansen Int [Internet]. 2014 [citado 2017 mar 22];39(2):19-26. Disponível em: http://www.ilsl.br/revista/download. php? id=imageBank/v39n2a03. pdf. 